\title{
云南大山包黑颈鹤日间越冬时间分配和活动节律
}

\author{
孔德军 ${ }^{1,2}$, 杨晓君 $1^{1, *}$, 钟兴耀 3 , 道美标 ${ }^{3}$, 朱 勇 $^{3}$ \\ (1. 中国科学院昆明动物研究所, 云南 昆明 $650223 ; 2$. 中国科学院研究生院, 北京 100049 ; \\ 3. 大山包黑颈鹤国家级自然保护区管理局, 云南 昭通 657000)
}

\begin{abstract}
摘要: 2006 年 10 月一2007 年 4 月采用瞬时扫描取样法对云南省昭通市大山包黑颈鹤国家级自然保护区内越 冬的黑颈鹤(Grus nigricollis)种群进行了越冬时间分配和日间活动节律的研究。取食是黑颈鹤越冬期间最主要的行 为, 占 $(53.05 \pm 4.93) \%$, 其次为警戒 $(18.75 \pm 2.65) \%$ 、搜寻 $(10.38 \pm 1.34) \%$ 和休整 $(10.32 \pm 4.93) \%$, 其余行为依次为: 行 走 $(4.90 \pm 1.59) \%$ 、飞行 $(1.70 \pm 0.38) \%$ 、其他 $(0.55 \pm 0.41) \%$ 、争斗 $(0.36 \pm 0.25) \%$ 。黑颈鹤的出飞时间与日出时间、飞 回时间与日落时间分别具有显著的正相关和负相关关系。越冬期间黑颈鹤日间各时间段(1 h)(09: 00-18：00)和 各月份间 $(2006.11-2007.03)$ 行为差异极显著 $(\mathrm{P}<0.05)$, 并呈现出上午和下午取食双峰、中午休息高峰的规律性变 化。气温高时黑颈鹤增加休整时间减少取食时间; 气温低时则减少休整时间增加取食时间。偏相关分析说明: 环 境温度独立地对越冬时间分配产生影响, 湿度通过温度对黑颈鹤越冬时间分配产生相反的影响。
\end{abstract}

关键词: 大山包; 黑颈鹤; 时间分配; 活动节律

中图分类号: Q959.726 文献标识码: A 文章编号: 0254-5853-(2008)02-0195-08

\section{Diurnal Time Budget and Behavior Rhythm of Wintering Black-necked Crane (Grus nigricollis) at Dashanbao in Yunnan}

\author{
KONG De-jun ${ }^{1,2}$, YANG Xiao-jun ${ }^{1,},{ }^{*}$ ZHONG Xing-yao ${ }^{3}$, DAO Mei-biao ${ }^{3}$, ZHU Yong $^{3}$ \\ (1. Kunming Institute of Zoology, the Chinese Academy of Sciences, Kunming Yunan 650223, China; \\ 2. Graduate University of the Chinese Academy of Sciences, Beijing, 100049, China; \\ 3. Administrative Bureau, Dashanbao National Nature Reserve, Zhaotong Yunnan 657000, China)
}

\begin{abstract}
From October 2006 to April 2007, wintering behaviors and the daily cycle of Black-necked Cranes (Grus nigricollis) were observed with instantaneous and scan sampling methods at Dashanbao National Nature Reserve, Zhaotong, Yunnan. During the wintering period, to meet wintering energy needs Black-necked Cranes spent most of their time feeding, which was about $53.05 \pm 4.93 \%$. Other behaviors such as vigilance, searching, maintenance, walking, flight, and fight were $18.75 \pm 2.65 \%, 10.38 \pm 1.34 \%, 10.32 \pm 4.93 \%, 4.90 \pm 1.59 \%, 1.70 \pm 0.38 \%, 0.55 \pm 0.41 \%, 0.36 \pm 0.25 \%$, respectively. Our results indicated that roost departure time had positive correlation with sunrise time, while roost arrival time had negative correlation with sunset time. One-Way ANOVA analysis showed that the eight wintering behaviors of Black-necked Cranes had significant differences between every one-hour period (09:00-18:00) and over the five month period (2006-11-2007-03). There were two feeding peaks in the morning and afternoon, with one maintenance peak at noon. Partial analyses showed that environmental temperature affected wintering behaviors independently, while environmental moisture had the opposite effect on behaviors dependent on environmental moisture. When the temperature was high, feeding was low and maintenance was high. When the temperature was low, maintenance was high and feeding was low.
\end{abstract}

Key words: Dashanbao; Black-necked Crane; Time budget; Behavior rhythm

黑颈鹤(Grus nigricollis)是世界上 15 种鹤中唯 种终生生活在高原上的鹤类, 同时也是科学发
现最晚的鹤类。主要分布在中国的青藏高原和云贵 高原，在国外仅不丹和印度的克什米尔有少量分布

收稿日期：2008-01-03; 接受日期：2008-03-07

基金项目：国家重点基础研究发展计划资助项目(2007CB411600); 中国科学院知识创新项目(KSCX2-SW-119); 国际鹤类基金会资助项目; 中 国科学院西部之光联合学者资助项目

*通讯作者(Corresponding author), E-mail: yangxj@mail.kiz.ac.cn

第一作者简介: 孔德军(1983-), 男, 硕士研究生, 主要从事鸟类行为生态学研究。 
( $\mathrm{Li}, 2005)$ 。根据 $\mathrm{Li} \& \mathrm{Li}(2005)$ 最新数据, 估计全 世界黑颈鹤的越冬种群数量大于 8000 只, 其中我 国西藏约 4300 只、云贵高原 3500 只左右、不丹约 400 只。由于其种群数量稀少而被列为国家 I 级重 点保护野生动物, 并被 IUCN 列为全球性易危物种 (Birdlife International, 2006) 和 CITES 附录 I 中 (Inskipp \& Gillett, 2005)。

活动节律与时间分配是动物行为学研究的重要 内容, 直接与动物的新陈代谢与能量需求相关 (Halle \& Stenaeth, 2000), 而这些又随着环境条件的 变化而变化(Orians，1961; Verbeek，1964，1972; Caraco, 1979b)。众多学者对鸟类的研究表明, 食物 是影响鸟类时间分配的主要因素之一 (Caraco, 1979b; Hixon, 1983; Davies \& Lundberg, 1985), 食物 密度影响鸟类的取食强度而不是取食时间, 当栖息 地内食物密度较高时, 取食强度较高, 鸟类的警戒 力也维持一个较高水平(Enoksson, 1990)。温度与鸟 类的能量分配直接相关, 从而影响鸟类的时间分配 (Kendeigh, 1949; Davis, 1955; Verbeek, 1964, 1972; Caraco, 1979b)。由于黑颈鹤被发现较晚, 并且其生 存环境海拔高、温度低, 长期以来科学家对其研究 较少。上个世纪 $80 、 90$ 年代主要集中在对黑颈鹤 越冬地的考察、种群数量的统计、越冬生态的初步 研究上(Wang et al, 1989; Zhang \& Luo, 1991; Li \& Ma, 1992; Yu et al, 1993; Chen, 1994, 1997; Li et al, 1997); 21 世纪以来科学家开始对黑颈鹤进行了个 体行为、食性分析、繁殖行为和卫星跟踪的迁徙研 究(Li \& Ma, 2000; Mary \& Li, 2002; Yang et al, 2006; Gao et al, 2007; Yang et al, 2007)。对于黑颈鹤越冬 行为时间分配的观察目前仅见有 $\mathrm{Li} \& \mathrm{Ma}$ (1992)在 贵州草海的研究, 其研究主要对集群鹤和家庭鹤的 时间分配和集群利益进行了比较和分析。而对大山 包越冬的黑颈鹤时间分配和日间活动节律尚无报 道。2006 年 10 月至 2007 年 4 月在大山包黑颈鹤国 家级自然保护区, 我们对黑颈鹤的越冬行为时间分 配和活动节律性进行了观察, 并拟从温度和湿度入 手来探讨黑颈鹤在高寒地区的生存策略。

\section{1 研究区域和方法}

\section{1 研究区域}

保护区位于云南省昭通市昭阳区西部, 其范围 包括大山包乡全境 $\left(\mathrm{N} 27^{\circ} 18^{\prime} 38^{\prime \prime}-27^{\circ} 29^{\prime} 15^{\prime \prime}\right.$, $\left.\mathrm{E} 103^{\circ} 14^{\prime} 55^{\prime \prime}-103^{\circ} 23^{\prime} 49^{\prime \prime}\right)$, 总面积为 $19200 \mathrm{~km}^{2}$ 。
保护区海拔在 3000-3 200 m 之间, 境内山峰绵延 起伏、山体浑圆、坡度平缓、相对高差较小, 平均 为 $50-100 \mathrm{~m}$; 谷地为开阔的亚高山沼泽化草甸、 湿地及高原湖泊。保护区气候冬寒夏凉、气温较低, 年平均气温为 $6.2^{\circ} \mathrm{C}, 1$ 月均温为 $-1^{\circ} \mathrm{C}, 7$ 月均温 $12^{\circ} \mathrm{C}$, 年降水量 $1165 \mathrm{~mm}$, 年均积雪日数 34.6 天, 平均无霜期 134 天。据保护区多年的观察资料表明, 黑颈鹤每年到大山包保护区越冬数量是初期较大, 中期有所下降, 并较长时间维持在 800-1000 只左 右, 至越冬末期又增至初期的 1200 余只(Zhong \& Dao, 2005)。

观察地点选择在保护区中最大的一个夜栖地 一大海子的周围, 2006-2007 年观察到的越冬黑 颈鹤有 500-800 只, 占保护区当年黑颈鹤总数量 的 $1 / 2-2 / 3$ 。保护区内伴随黑颈鹤越冬的常见水禽 有灰鹤(Grus grus)、苍路 (Ardea cinerea)、白鹭 (Egretta garzetta)、大白鹭(Casmerodius albus)、池 鹭(Ardeola bacchus)、斑头雁(Anser indicus)、赤麻 鸭 (Tadorna ferruginea) 、绿头鸭 (Anas platyrhynchos)、绿翅鸭 (Anas crecca)、风头潜鸭 (Aythya fuligula)、普通秋沙鸭(Mergus merganser)等。 其中灰鹤常与黑颈鹤混群活动, 其余水禽多不与黑 颈鹤混群, 但绿头鸭、绿翅鸭在人工投食时会与黑 颈鹤争食。

\section{2 调查方法}

黑颈鹤在大山包保护区内的越冬时间为每年的 10 月底一 11 月初至次年的 4 月末一 5 月初(Hu et al, 2002), 我们于 2006 年 10 月在大山包自然保护区最 大的一个夜栖地一大海子的周围, 选定观察地点 和设立 2 条固定调查路线, 并进行预观察, 熟悉观 察方法和黑颈鹤的越冬行为。观察点选择在大海子 夜栖地边的观鹤屋; 调查路线则是避开了保护区内 人为干扰较大的大路, 而选择了小路和山脊横梁, 两条固定样线早晚可以观察到该夜栖地内的所有 黑颈鹤, 白天可以观察到绝大多数的黑颈鹤和保护 区内的各种栖息地类型。正式观察从 2006 年 11 月 2 日开始至 2007 年 3 月 28 日结束。选取晴天进行 观察, 由于大山包为高寒山区, 气候变化复杂, 因 此观察时如果发生起雾、下雪等影响观察效果的天 气变化, 则按小时停止该时段的观察, 当天气情况 允许时再继续当日的观察。每天的早 06 : $00-09$ : 00)、晚(18：00-19：00)在观察点统计黑颈鹤的起 飞时间和返回时间。09：00-18：00 沿固定样线调 
查, 利用 Swarovski Habicht 10×42 双筒望远镜采用 瞬时扫描法(Martin \& Bateson, 1993)对遇见的可准 确鉴定行为的黑颈鹤群体进行 5 次瞬时扫描, 对无 法准确鉴定其行为的群体不作行为观察。由于黑颈 鹤行为变化频率较高, 且每次发生的行为持续时间 都小于 $55 \mathrm{~s}$ (Yang et al, 2007), 所以本次研究每两次 扫描之间时间间隔定为 $1 \mathrm{~min}$, 每次扫描持续时间在 $30 \mathrm{~s}$ 以内。记录黑颈鹤群体大小、时间、地点、生 境和个体行为。考虑到黑颈鹤直接活动在地面, 并 在土地浅层取食, 所以本文采用地面温度和地面湿
度, 即扫描结束后将温湿度计置于与鹤群所处栖息 地同质的地面上直接测量。同时记录当时的天气状 况, 并通过 GARMIN GPS12 获得当天的日出日落 时间。本文将能见度小于 $10 \mathrm{~m}$ 、降雪深度大于 $5 \mathrm{~cm}$ 的天气状况分别定义为大雾和大雪。

行为定义: 将黑颈鹤的越冬行为分为取食 (feeding)、搜寻(searching)、警戒(vigilance)、争斗 (fight)、行走(walk)、飞行(flight)、休整(maintenance) 和其他(others) 8 种，其行为描述见表 1 。

调查期间，黑颈鹤的起飞时间共统计 $31 \mathrm{~d}$ ，黑

表 1 云南昭通大山包黑颈鹤越冬行为描述

Tab.1 Description of wintering behaviors of Black-necked Cranes at Dashanbao, Yunnan, China

\begin{tabular}{ll}
\hline 行为类型Behavior types & \multicolumn{1}{c}{ 行为描述Behavior description } \\
\hline 取食Feeding & 与取食有关的探取、食物处理 (甩动、豚碎等)、吞咽的过程; 同时还包括发生频次很少的成 \\
搜寻Searching & 低头行走 \\
警戒Vigilance & 举头环视、扬头走动、远眺等 \\
争斗Fight & 仪式化威胁和直接争斗 \\
行走Walk & 非搜寻走动之外的行走 \\
飞行Flight & 飞行 \\
休整Maintenance & 理羽、抖羽、振翅、休息和洗澡等 \\
其他Others & 鸣叫、跳舞、追逐玩要和饮水 \\
\hline
\end{tabular}

颈鹤返回时间共记录 $22 \mathrm{~d}$ 。日间行为活动观察共进 行了 $62 \mathrm{~d}$, 累计 $351 \mathrm{~h}$, 共扫描 2517 次, 收集到 505 群次, 38793 只次的行为扫描数据。

\section{3 数据分析}

所有数据均输入计算机中, 分别统计黑颈鹤的 活动规律和时间分配等。将温度按每 $10^{\circ} \mathrm{C}$ 一个等级 分为: $t \leqslant 10^{\circ} \mathrm{C} 、 10^{\circ} \mathrm{C}<t \leqslant 20^{\circ} \mathrm{C} 、 20^{\circ} \mathrm{C}<t \leqslant 30^{\circ} \mathrm{C}$ 和 $t>30{ }^{\circ} \mathrm{C}$ 四级, 将湿度按每 $30 \%$ 一个等级分为: $\mathrm{m} \leqslant 30 \% 、 30 \%<\mathrm{m} \leqslant 60 \%$ 和 $\mathrm{m}>60 \%$ 三个等级。采 用 Pearson 相关性分析分析出飞时间与日出时间、 返回时间与日落时间的关系。用单因素方差分析 (One-Way ANOVA)分别分析天气状况(晴、雾、大 风)对飞出时间的影响、日间各时间段(1h)和各月份 间的行为差异。应用非参数多个独立样本检验 (K-Independent samples Test)检验温度和湿度对越 冬行为的影响。考虑到环境温度和湿度之间关系较 为复杂, 可能存在交互影响, 利用偏相关分析(partial) 对环境温度和湿度中一种因子在控制和不控制的 条件下, 另外一种因子对黑颈鹤行为时间分配的影 响是否一致进行检验。所有数据分析全部在 SPSS 13.0 软件中进行。

\section{2 结 果}

\section{1 日活动节律}

黑颈鹤在一天之内的几个主要行为具有较明显 的节律性: 其中取食、警戒、搜寻行为具明显的早、 晚两个高峰, 中午 13:00-4:00 最低, 只是搜寻的 最低峰要比取食晚一个小时; 而休整行为正好相 反; 另外几种行为由于所占比例较少, 节律性表现 不明显(图 1)。单因素方差分析表明: 黑颈鹤在一天 的各个时段内(以 $1 \mathrm{~h}$ 为单位)取食、警戒、争斗、飞 行、休整和其它行为时间分配存在极显著的差异 $\left(F_{9}=2.616-13.557, P=0.000-0.004\right)$ 。

\section{2 越冬时间分配}

在整个越冬季节，取食行为占了黑颈鹤日间时 间分配的一半以上 $[(53.05 \pm 4.93) \%]$, 与取食相关的 搜寻为 $(10.38 \pm 1.34) \%$, 同时警戒和休整也占有很大 的比例, 分别为 $(18.75 \pm 2.65) \%$ 和 $(10.32 \pm 4.93) \%$ 。而 另外 4 种行为: 行走、飞行、争斗和其他在黑颈鹤 的越冬期所占比例较小依次为 $(4.90 \pm 1.59) \%$ 、 $(1.70 \pm 0.38) \%$ 、 $(0.36 \pm 0.25) \%$ 和 $(0.55 \pm 0.41) \%$ 。单因 素方差分析表明: 黑颈鹤的取食、警戒、休整、行 走、其他行为具有极显著的月变化 $\left(F_{4}=3.544-\right.$ 


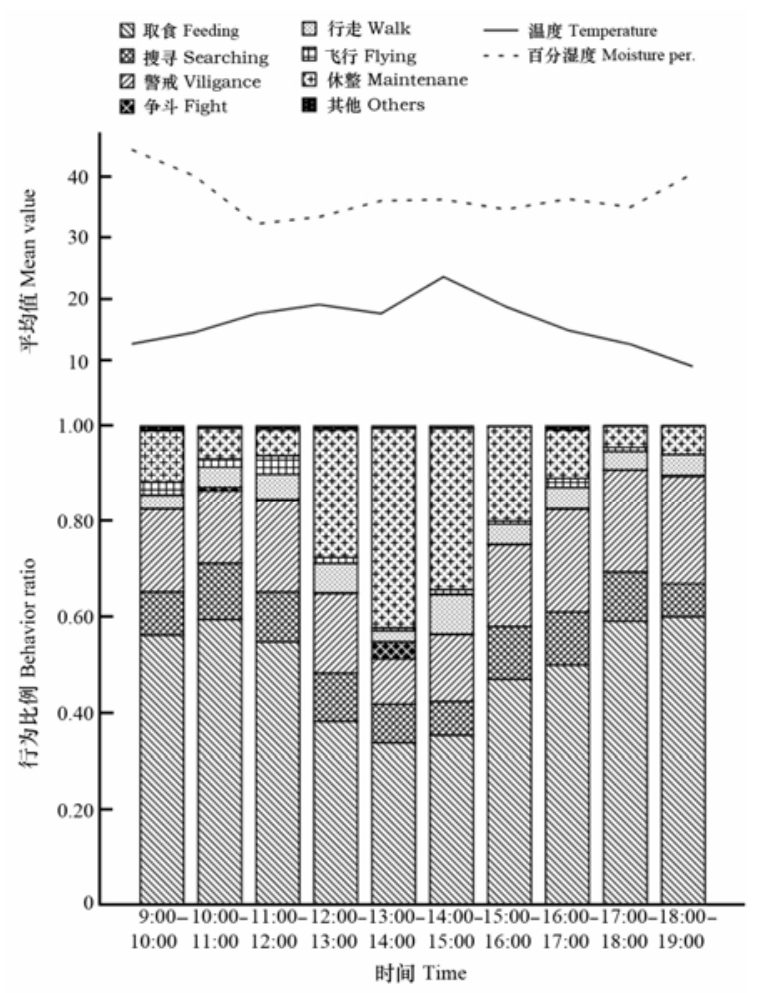

图 1 云南昭通大山包越冬黑颈鹤日间不同时间段的行为、 温度和湿度变化

Fig. 1 Behaviors, temperature and moisture changes during different one-hour periods of wintering Black-necked Cranes at Dashanbao, Yunnan, China

$11.131, P=0.000-0.007)$, 争斗行为具有显著的月 变化 $\left(F_{4}=2.684, P=0.030\right)$, 而搜寻和飞行的月间 差异不显著 $\left(F_{4}=0.584-2.232, P=0.063-0.675\right)$ (图 2)。

\section{3 温度对行为的影响}

随温度的升高取食行为呈下降趋势, 而休整行 为却呈现出上升趋势(图 3); 搜寻和警戒行为大体趋 势也是随着温度的升高而降低的, 只是搜寻在 10 ${ }^{\circ} \mathrm{C}<t \leqslant 20^{\circ} \mathrm{C}$ 温度范围内有一个上升, 警戒在 $t>30$ ${ }^{\circ} \mathrm{C}$ 范围内有一个上升。另外几种行为随温度的变化 趋势不明显(图 3)。黑颈鹤月份间行为变化随月份间 温度的变化也具有相同的趋势(图 2)。应用非参数多 个独立样本检验, 结果表明: 不同温度等级下黑颈 鹤的取食、休整、搜寻、警戒和行走等主要行为均 具有显著的差异(表 2)。偏相关分析结果说明: 在控 制和不控制湿度两种条件下, 温度对黑颈鹤行为时 间分配的影响结果前后一致(表 3)。

\section{4 湿度对行为的影响}

从观察结果上看, 黑颈鹤的取食活动随着湿度 的增加而增加, 而休整行为呈明显下降趋势; 警戒

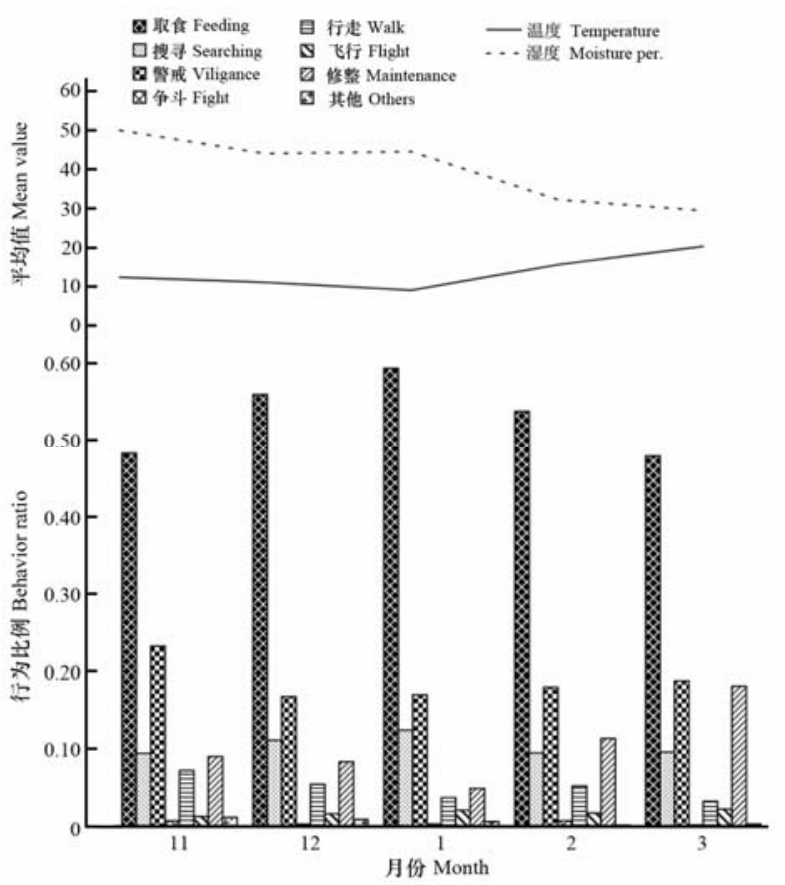

图 2 云南昭通大山包越冬黑颈鹤月间行为、温度 和湿度变化

Fig. 2 Behaviors, temperature and moisture changes among different months of wintering Black-necked Cranes at Dashanbao, Yunnan, China

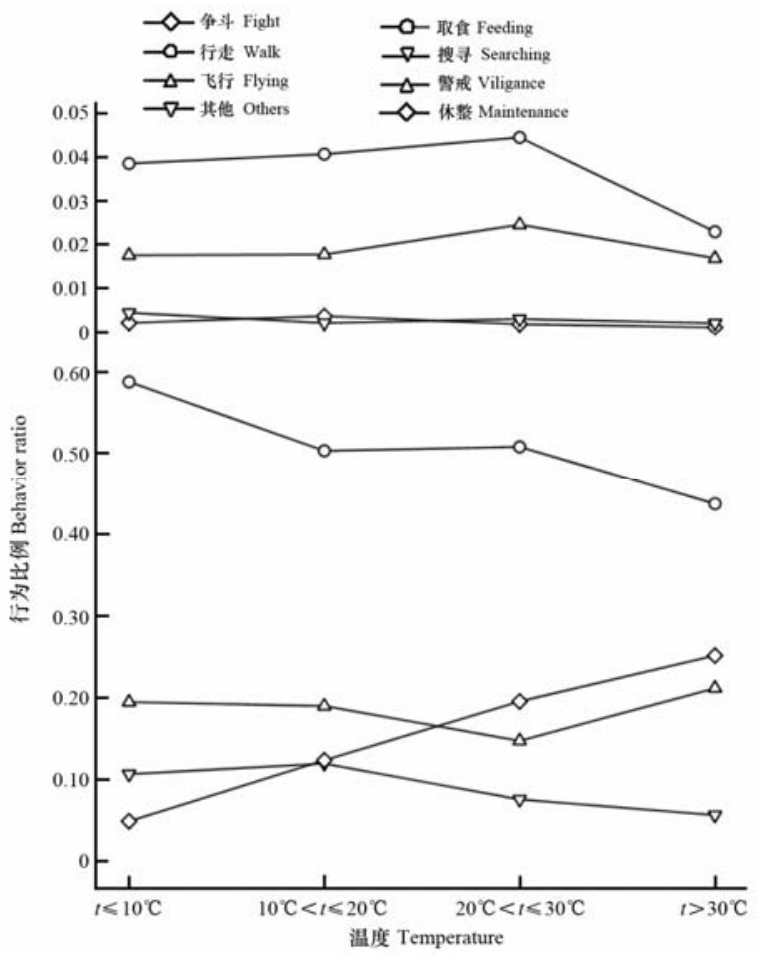

图 3 云南昭通大山包黑颈鹤越冬行为随温度的变化

Fig. 3 Behavior changes with temperature of wintering Black-necked Cranes at Dashanbao, Yunnan, China 
行为变化趋势不明显。应用非参数多个独立样本检 验表明, 湿度仅对黑颈鹤越冬行为中的取食、警戒 和休整行为影响差异显著 $(P<0.05)$, 而对其他 5 种 行为无显著影响 (表 2)。偏相关分析显示: 在不控制 温度条件下, 取食、休整和其他 3 种行为随湿度的 变化差异显著; 但是, 在控制温度条件下黑颈鹤的 所有行为差异都不显著(表 3)。

\section{5 日活动时间}

黑颈鹤通常在早上飞离夜栖地, 部分个体中午 返回夜栖地休息, 然后再出去受食, 傍晚则全体返 回夜栖地休息。天气晴好时, 黑颈鹤平均出飞时间 为 $07: 18 \pm 0: 15(06: 57-07: 46, n=31)$ 。据观察, 大雾严重影响了黑颈鹤的外出受食, 在大雾的时候 黑颈鹤推迟到 09: 18 $\pm 01: 39(n=8)$ 才飞出, 最晚可 到 11：15 或雾散之后。如果一天大雾不散, 部分 黑颈鹤则整天停留在夜栖地中。单因素方差分析表 明, 天气状况对黑颈鹤的出飞时间具有极显著性的 影响 $\left(F_{4}=19.912, P=0.000\right)$, Pearson 相关性分析显 示, 黑颈鹤的出飞时间与日出时间具极显著的正相
关关系 $(r=0.847, P=0.000, n=31)$ (图 2)。黑颈鹤傍晚 返回夜栖地的时间平均为 $18: 01 \pm 00: 32(16$ : 33-18: 33, $n=22)$ 。Person 相关分析表明黑颈鹤傍 晚返回夜栖地的时间与日落具极显著的负相关关 系 $(r=-0.715, P=0.000, n=22)$ 。

\section{3 讨 论}

\section{1 日活动时间}

最早提出时间和能量分配概念的 Orians(1961) 指出每种鸟花费在每种行为上的时间和能量都肯 定影响鸟的生存。所以，鸟类的时间分配不仅是对 当地环境条件的一种适应, 同时也是影响动物活动 的全部因素的综合表现(Yang \& Yang, 1996)。每一 个物种都有适于自己的时间分配和行为节律, 有最 适时间分配和行为节律的个体在自然选择中是有 利的(Verbeek, 1972; Coroco, 1979a)。本研究中，黑 颈鹤在越冬期间取食行为占日活动的 50\%以上，其 次是警戒和休整行为，与取食相关的搜寻行为也占 了很大的比例, 这与贵州草海越冬的黑颈鹤(Li \&

表 2 云南昭通大山包黑颈鹤越冬行为受温度和湿度影响的多个独立样本的非参数检验结果

Tab. 2 Nonparametric K-Independent samples Test of wintering behaviors of Black-necked Cranes affected by temperature and moisture at Dashanbao, Yunnan, China

\begin{tabular}{|c|c|c|c|c|c|c|c|c|c|}
\hline $\begin{array}{c}\text { 环境因子 } \\
\text { Environmental factor }\end{array}$ & $\begin{array}{c}\text { 统计参数 } \\
\text { Statistical parameter }\end{array}$ & $\begin{array}{c}\text { 取食 } \\
\text { Feeding }\end{array}$ & $\begin{array}{c}\text { 搜寻 } \\
\text { Searching } \\
\end{array}$ & $\begin{array}{c}\text { 警戒 } \\
\text { Viligance }\end{array}$ & $\begin{array}{l}\text { 争斗 } \\
\text { Fight }\end{array}$ & $\begin{array}{l}\text { 行走 } \\
\text { Walk } \\
\end{array}$ & $\begin{array}{c}\text { 飞行 } \\
\text { Flight } \\
\end{array}$ & $\begin{array}{c}\text { 休整 } \\
\text { Maintenance } \\
\end{array}$ & $\begin{array}{l}\text { 其他 } \\
\text { Others }\end{array}$ \\
\hline \multirow{4}{*}{ 温度 Temperature } & 样本量 N & 2006 & 2006 & 2006 & 2006 & 2006 & 2006 & 2006 & 2006 \\
\hline & 卡方值 Chi-Square & 13.449 & 17.919 & 10.801 & 3.761 & 19.839 & 2.702 & 96.082 & 20.807 \\
\hline & 自由度 $\mathrm{df}$ & 3 & 3 & 3 & 3 & 3 & 3 & 3 & 3 \\
\hline & 显著性 Significance & $0.004^{* *}$ & $0.000^{* *}$ & $0.013^{*}$ & 0.288 & $0.000^{* *}$ & 0.440 & $0.000^{* *}$ & $0.000^{* *}$ \\
\hline \multirow{4}{*}{ 湿度 Moisture } & 样本量 N & 2006 & 2006 & 2006 & 2006 & 2006 & 2006 & 2006 & 2006 \\
\hline & 卡方值 Chi-Square & 21.112 & 2.960 & 7.046 & 3.731 & 0.961 & 2.082 & 37.549 & 0.398 \\
\hline & 自由度 df & 2 & 2 & 2 & 2 & 2 & 2 & 2 & 2 \\
\hline & 显著性 Significance & $0.000^{* *}$ & 0.228 & $0.030^{*}$ & 0.155 & 0.619 & 0.353 & $0.000^{* *}$ & 0.819 \\
\hline
\end{tabular}

${ }^{*} \mathrm{P}<0.05 ;{ }^{* *} \mathrm{P}<0.01$.

表 3 两种条件下温度和湿度对云南昭通大山包黑颈鹤越冬行为影响的偏相关分析结果

Tab. 3 Partial Analysis of wintering behaviors of Black-necked Cranes affected by temperature and moisture under two conditions at Dashanbao, Yunnan, China

\begin{tabular}{|c|c|c|c|c|c|c|c|c|c|}
\hline $\begin{array}{l}\text { 环境因子效应 } \\
\text { Effect of environmental } \\
\text { factor }\end{array}$ & $\begin{array}{c}\text { 不同条件 } \\
\text { Different condition }\end{array}$ & $\begin{array}{c}\text { 取食 } \\
\text { Feeding }\end{array}$ & $\begin{array}{c}\text { 搜寻 } \\
\text { Searching }\end{array}$ & $\begin{array}{c}\text { 警戒 } \\
\text { Vigilance }\end{array}$ & $\begin{array}{l}\text { 争斗 } \\
\text { Fight }\end{array}$ & $\begin{array}{l}\text { 行走 } \\
\text { Walk }\end{array}$ & $\begin{array}{l}\text { 飞行 } \\
\text { Flight }\end{array}$ & $\begin{array}{c}\text { 休整 } \\
\text { Maintenance }\end{array}$ & $\begin{array}{l}\text { 其他 } \\
\text { Others }\end{array}$ \\
\hline 温度影响 & $\begin{array}{l}\text { 不控制湿度 } \\
\text { Moisture no controled }\end{array}$ & $0.000^{* *}$ & $0.001^{* *}$ & $0.036^{*}$ & 0.723 & 0.759 & 0.252 & $0.000^{* *}$ & 0.167 \\
\hline Temperature effect & $\begin{array}{l}\text { 控制湿度 } \\
\text { Miosture controled }\end{array}$ & $0.001^{* *}$ & $0.000^{* *}$ & $0.020^{*}$ & 0.352 & 0.981 & 0.332 & $0.000^{* *}$ & 0.991 \\
\hline 湿度影响 & $\begin{array}{l}\text { 不控制温度 } \\
\text { Temprature no controled }\end{array}$ & $0.000^{* *}$ & 0.527 & 0.640 & 0.566 & 0.650 & 0.533 & $0.000^{* *}$ & $0.031^{*}$ \\
\hline Moisture effect & $\begin{array}{l}\text { 控制温度 } \\
\text { Temperature controled }\end{array}$ & 0.052 & 0.1 & 0.263 & 0.301 & 0.737 & 0.893 & 0.441 & 0.096 \\
\hline
\end{tabular}

${ }^{*} P<0.05 ;{ }^{* *} P<0.01$. 
Ma, 1992)、西班牙越冬的灰鹤(Grus grus)(Alonso \& Alonso, 1992; Alonso et al, 2004)、鄱阳湖越冬的白 鹤(Grus leucogeranus)(Wu, 2005)和盐城越冬的丹顶 鹤(Grus japonensis)(Tian et al, 2006)的研究结果相 似, 而与笼养越冬白鹤(Tian et al, 2005)的行为时间 分配相反。这与笼养状态下食物丰富、安全有一定 的保障, 而野外条件下食物相对来说比较缺乏、潜 在危险较多, 鸟类必须花费较多的时间来寻找并取 得食物和防御天敌有关。

与贵州草海越冬黑颈鹤相比, 大山包越冬的黑 颈鹤每天花费在搜寻和摄取食物上的时间 (63.43\%), 低于草海越冬黑颈鹤搜寻和取食行为的 时间(家庭鹤 72.30\%; 集群鹤 79.34\%), 而大山包黑 颈鹤越冬警戒行为比例(18.75\%)高于草海越冬黑颈 鹤(集群鹤 $12.59 \%$; 家庭鹤 16.52\%)。产生这种情况 的原因可能有: (1)大山包自然保护区黑颈鹤自然食 物资源较为贫乏, 保护区常在一定时间内人工补充 食物, 在一定程度上增加了食物密度, 造成黑颈鹤 的取食时间减少; (2)草海黑颈鹤活动地区多为沼泽 生境, 人类及牲畜都较难到达, 而大山包的黑颈鹤 多在耕地和草地活动, 人畜都可轻易到达, 观察期 间曾发生过多次人以及猪、狗追赶黑颈鹤的现象, 在过去甚至还发生过狗咬伤、咬死黑颈鹤的情况, 所以对黑颈鹤的干扰较大。

大山包越冬黑颈鹤花费在休息、理羽和维持自 身清洁上的时间比例 $(10.32 \%)$, 高于草海越冬黑颈 鹤的时间比例(家庭鹤 7.94\%和集群鹤 5.58\%)。这除 与人工投喂食物减少了大山包黑颈鹤的受食时间 有关外, 还可能与大山包水域面积有限, 只有几个 较为集中的水库, 在中午的时候黑颈鹤会飞回夜栖 地处集中洗澡、饮水和休息有关。在盐城国家级自 然保护区越冬的丹顶鹤也有此集中休息的习性(Lü \& Chen, 2006); 而草海水域面积较大, 黑颈鹤没有 这种集中休息行为, 故休整行为所占比例较低。

\section{2 日间节律}

越冬黑颈鹤的行为活动具有明显的昼夜节律, 与同属的越冬灰鹤具有相似的活动节律(Alonso \& Alonso, 1992)。黑颈鹤的出飞时间与每日的日出时 间紧密相连(图 4), 这与笼养大紫胸鹦武(Psittacula derbiana)一样(Yang et al, 1998)。黑颈鹤的傍晚返回 时间同日落时间呈负相关可能是由于白天的时间 长短随季节变化而变化造成的。白天日时间短时, 由于日时间缩短造成黑颈鹤日间受食不足, 为了弥

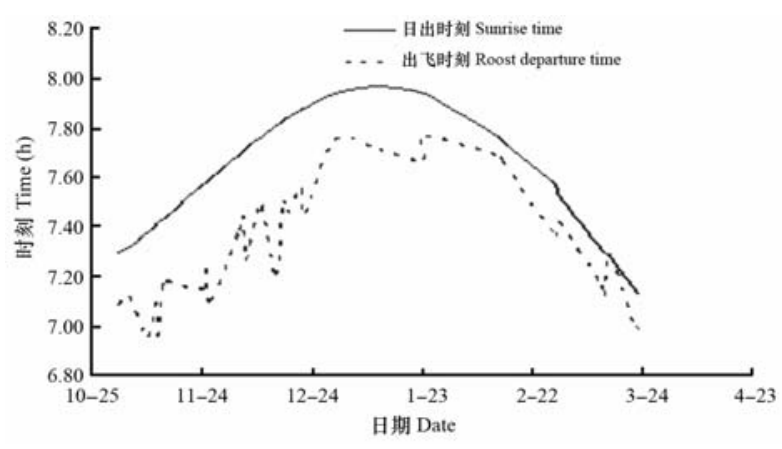

图 4 云南昭通大山包越冬黑颈鹤出飞时间与日出时间

Fig. 4 Roost departure time of wintering Black-necked Cranes and sunrise time at Dashanbao, Yunnan, China

补取食量的不足黑颈鹤不得不延迟飞回时间, 相似 的现象也出现于丹顶鹤越冬行为中 (Lü \& Chen, 2006)。另外, 黑颈鹤在大雾天气明显延后了出飞时 间, 这与丹顶鹤在大雾天气时的表现一致(Lü \& Chen, 2006)。但是, 连续几天大雾后黑颈鹤会调整 飞出时间, 2006 年 11 月 27 日至 2006 年 12 月 3 日 期间连续大雾, 能见度小于 $10 \mathrm{~m}$, 黑颈鹤几乎全天 都未飞出, 仅在夜栖地周围沼泽、山坡受食, 而在 2006 年 12 月 3 日，08：00 时黑颈鹤便飞出受食以 补充几日内的能量消耗。

黑颈鹤日间的行为变化节律与日间行为的月份 变化, 都与环境因子中的温度和湿度紧密相关(图 3、4)。上午和下午温度较低, 中午 13: $00-15$ : 00 温度最高, 黑颈鹤行为也表现出相应的变化: 上 午和下午取食行为较高, 休整行为较低; 而中午休 整行为最高, 取食行为最低。这可能是因为上午和 下午温度较低, 能量流失较重(Kendeigh, 1949; Davis, 1955), 黑颈鹤为了满足自身能量的需求必须 外出受食, 从而减少了休息时间; 中午温度较高则 可以减少能量的流失, 进而吸收太阳光的能量以保 持体温, 这样相应地就减少了受食时间、增加了休 整时间。黑颈鹤的取食行为上午、下午双峰和休整 行为中午高峰的这种行为模式与越冬灰鹤相一致 (Alonso \& Alonso, 1992)。这种取食行为与温度变 化呈相反的趋势在很多研究中都得到证明(Verbeek, 1964, 1972; Caraco, 1979b)。同时, 在观察过程中还 发现黑颈鹤在大风低温时, 多迎风取食, 而在无风 晴朗天气时多背向太阳取食, 与 Lü \& Chen (2006) 对盐城越冬丹顶鹤的观察相同。大风时迎风取食除 了为了保持身体平衡外, 还可能是为了避免由于风 吹翻羽毛导致皮肤裸露所造成的能量损失, 而无风 
时背向太阳取食则是为了利用尾部黑色羽毛更好 的吸收阳光。这一切可能是黑颈鹤为了在这种高寒 地区保持体温和维持能量所采取的措施。警戒行为 的中午低谷是由于黑颈鹤中午集中返回夜栖地休 息, 大部分个体都在休息而只有几个个体负责警 戒, 所以黑颈鹤警戒行为在中午下降而休整行为中 午达到峰值。搜寻行为变化同取食变化基本相同, 因为搜寻是同取食相伴发生的, 只是黑颈鹤中午返 回以后部分黑颈鹤还会一直搜寻受食, 所以搜寻行 为在 14: 00-15: 00 才达到最低点。其他行为在 13：00-14：00 达到最高是由于黑颈鹤在夜栖地重 新集合, 黑颈鹤间的社会行为如: 鸣叫、追逐嬉戏 和饮水个体增多的缘故。

\section{3 环境因子影响}

在温度低的月份, 黑颈鹤增加了取食、搜寻和 警戒时间比例、减少了休整时间; 当温度升高时, 黑颈鹤增加了休整时间而降低了取食和警戒行为 的时间, 这与越冬大铇(Otis tarda)(Sun et al, 2006) 和笼养白鹤(Tian et al, 2005)的研究结果正好相反。 这与笼养状态下食物资源充足有密切关系, 还可能 与黑颈鹤生活在高原, 适应寒冷气候, 而大铇、白 鹤等适应平原环境有关。

非参数多个独立样本检验表明，黑颈鹤时间分 配是受环境温度和湿度影响的。进一步的偏相关分 析显示: 当环境湿度在控制和不控制的条件下, 温 度对黑颈鹤行为时间分配的结果是一致的(表 3), 说 明温度对黑颈鹤行为产生的影响不受环境湿度的 影响, 即温度对黑颈鹤行为的影响是独立起作用 的。而湿度对行为的影响则是通过温度来起作用 的, 因为当在控制环境温度时, 本来受湿度影响显
著的行为开始变的不再显著, 并且所有行为差异都 不显著(表 3)。可见, 黑颈鹤在高海拔寒冷地区越冬 所采用的越冬策略是高温时增加休息时间、低温时 增加取食时间，黑颈鹤在取食与休整之间所达到的 这种权衡是对生存有利的, 这种生存策略是黑颈鹤 为适应高原特殊的环境条件而发展起来的。

\section{4 保护建议}

黑颈鹤行为时间分配是随着环境温度的变化而 变化的, 在温度较低的时候增加取食时间以维持自 身能量需求; 保护区内的猪、狗的活动严重干扰了 黑颈鹤的正常活动。针对以上问题特提出以下保护 建议: (1)为避免寒冷季节由于食物的减少而造成的 黑颈鹤死亡, 建议在气温较低月份尤其是 12 月、1 月和大雾天气时, 适当的人工投放一些饲料, 如玉 米(Zea mays)、马铃薯(Solanum tuberosum)等; (2) 改变传统耕种方式, 提倡秋收后百姓不要捡拾田地 里残留的马铃薯、蔓荠(Rorippa indica)、燕麦(Avena sativa) 和苦菾麦(Fagopyrum tataricum), 为黑颈鹤提 供一些越冬食物; (3)加强保护区管理避免人畜直接 闯入黑颈鹤夜栖地而引起的外界干扰; (4)促进社区 发展、提高百姓的保护意识从而更好、更根本的保 护黑颈鹤。

致谢：此次野外工作得到了云南省大山包黑颈 鹤国家级自然保护区管理局的计扬善、陈俊、冯大 林、罗顺义、陈波、藏庆光、孟太国等同志的无私 帮助, 在此致以诚挚的谢意! 感谢师兄伍和启博士 和刘强博士在野外实验和论文修改方面给予的建 议, 感谢师兄范朋飞博士在实验设计和数据处理上 给予的帮助!

\section{参考文献:}

Alonso JC, Alonso JA. 1992. Daily activity and intake rate patterns of wintering common cranes (Grus grus) [J]. Ardea, 80:343-351.

Alonso JC, Bautista LM, Alonso JA. 2004. Family-based territoriality vs flocking in wintering common cranes (Grus grus) [J]. Journal of Avian Biology, 35:434-444.

BirdLife International. 2006. Grus nigricollis. In: IUCN 2007. 2007 IUCN Red List of Threatened Species. <www.iucnredlist.org>.

Caraco T. 1979a. Time budget and group size: A theory [J]. Ecology, 60(3): 611-617.

Caraco T. 1979b. Time budget and grope size: A test of theory [J]. Ecology, 60(3): 618-627.

Chen XP. 1994. Black-necked Cranes and overwintering places investigation on the Yunnan and Guizhou Plateau [J]. Yunnan Geographic Environment Research, 6(1): 84-87.[陈晓平. 1994. 滇东北

一黔西北高原黑颈鹤及其主要越冬地考察. 云南地理环境研究, 6(1): 84-87.]

Chen ZD. 1997. Wintering ecology and environmental effect observation [J]. Biosphere Reserve of China, 4: 1-17. [陈祯德. 1997. 黑颈鹤越冬习性 与环境影响观察. 中国生物圈保护区, 4: 1-17.]

Davies NB, Lundberg A. 1985. The influence of food on time budgets and time of breeding of the Dunnock (Purnella modularis) [J]. Ibis, 127: 100-110.

Davis EAJr. 1955. Seasonal changes in the energy balance of the English Sparrow [J]. Auk, 72: 385-411.

Enoksson B. 1990. Time budgets of Nuthatches (Sitta europaea) with supply entary food [J]. Ibis, 132: 375-583.

Gao LB, Qian FW, Yang XJ, Wu HQ, Li FS. 2007. Satellite tracking on the migratory routes of wintering Black-necked Cranes at Dashanbao in 
Yunnan. Zoological Research, 28(4):353-361. [高立波, 钱法文, 杨晓 君, 伍和启, 李凤山. 2007. 云南大山包越冬黑颈鹤迁徙路线的卫星 跟踪. 动物学研究, 28(4):353-361.]

Halle S, Stenaeth NC. 2000. Activity Pattens in Small Mammals: An Ecological Approach [M]. New York: Springer-Verlag Berlin Heidelberge.

Hixon MA, Carpenter FL, Paton DC. 1983. Territory area, flower density, and time budgeting in hummingbirds: An experimental and theoretical analysis [J]. The American naturalist, 12(3): 366-391.

Hu JS, Wu JL, Dang CL, Zhong XY, Dao MB. 2002. A study on the population ecology of wintering Black-necked Cranes (Grus nigricollis) at Dashanbao Reserve, Zhaotong, Yunnan Province [J]. Journal of Yunnan University, 24(2): 140-143. [胡健生, 吴金亮, 党承林, 钟兴 耀, 道美标. 2002. 云南昭通大山包自然保护区越冬黑颈鹤种群生态 研究. 云南大学学报(自然科学版), 24(2): 140-143.]

Inskipp T, Gillett HJ. 2005. Checklist of CITES species and Annotated CITES Appendices and reservations. Compiled by UNEP-WCMC. CITES Secretariat, Geneva, Switzerland and UNEP-WCMC, Cambridge, UK.

Kendeigh SC. 1949. Effect of temperature and season in energy resources of the English Sparrow [J]. Auk, 66(2): 113-127.

Li FS, Ma JZ. 1992. Wintering time budget and benefit study between family and group Black-necked Cranes [J]. Chinese Wildlife, 3: 36-41,29. [李风山, 马建章. 1992. 越冬黑颈鹤的时间分配、家庭和集 群利益的研究. 野生动物, 3:36-41,29.]

Li FS, Ma JZ. 2000. Behavioral ecology of black-necked crane during winter at Caohai, Guizhou, China [J]. Acta Ecologica Sinica, 20(2): 293-298. [李风山, 马建章. 2000. 越冬期黑颈鹤个体行为生态的研究. 生态学报, 20(2): 293-298.]

Li FS, Nie H, Ye CH. 1997. Microscopic analysis on herbivorous diets of wintering Black-necked Cranes(Grus nigricollis) at Caohai, China [J]. Zool Res, 18(1): 51-57. [李凤山, 聂 卉, 叶长虹. 1997. 黑颈鹤越冬 期间植物性食物的纤维分析. 动物学研究, 18(1): 51-57.]

Li FS. 2005. Status and Conservation of Black-necked Cranes [A]. In: Li FS, Yang XJ, Yang F. Status and conservation of Black-necked Cranes on the Yunnan and Guizhou Plateau, People' Republic of China [M]. Kunming: Yannan Nationalities Publishing House, 44-56. [李凤山. 2005. 黑颈鹤的现状和保护. 见: 李风山, 杨晓君, 杨 芳. 云贵高 原黑颈鹤的现状和保护. 昆明: 云南民族出版社, 44-56.]

Li ZM, Li FS. 2005. Black-necked Crane Study [M]. Shanghai Technological and Educational Press.[李筑眉, 李凤山. 2005. 黑颈鹤 研究. 上海科技教育出版社.]

Lü SC, Chen WH. 2006. Influence of environmental factors in overwintering behavior of Red-crowned Crane [J]. Chinese Wildlife, 27(6): 18-20. [吕士成, 陈卫华. 2006. 环境因素对丹顶鹤越冬行为的 影响. 野生动物杂志, 27(6): 18-20.]

Martin P, Bateson P. 1993. Measuring behavior: An introductory guide, 2nd ed $[\mathrm{M}]$. Cambridge, UK: Cambridge University Press.

Mary AB, Li FS. 2002. Effects of farming practices in Tibet on wintering Black-necked Cranes(Grus nigricollis) diet and food availability [J]. Biodiversity Science, 10(4): 393-398.[Mary AB, 李凤山. 2002. 农业耕 作活动对西藏越冬黑颈鹤食性及食物可获得性的影响. 生物多样 性, 10(4): 393-398.]

Orians GH. 1961. The ecology of blackbird (Agelaius) social systems [J]. Ecological Monographs, 31(3): 285-312.

Sun YF, Li SP, Li JY, Wu YF, Li JP. 2006. Time budget and activity rhythm of wild Great Bustard in winter [J]. Frontiers of Biology in China, 1(4): 443-447.

Tian XH, He XB, Zhou YR, Li XM. 2005. Observations on wintering behavior of captive Siberian Cranes at Harbin Zoo [A]. In: Wang QS, Li FS. Crane Research in China [M]. Kunming: Yunnan Educational Publishing House, 196-200. [田秀华, 何相宝, 周钲芯, 李晓敏. 2005. 笼养白鹤越冬行为的初步观察与分析. 见: 王岐山, 李风山. 中国鹤 类研究. 昆明: 云南教育出版社, 196-200.]

Tian XH, Shi QH, Lü SC, Li JB. 2006. Daytime budget of quasi-wild Grus japonensis in its wintering [J]. Chinese Journal of Ecology, 25(9): 1077-1081. [田秀华, 石全华, 吕士成, 李金邦. 2006. 半散放丹顶鹤 越冬日活动行为. 生态学杂志, 25(9): 1077-1081.]

Verbeek NAM. 1964. A time and energy budget study of the Brewer Blackbird [J]. The Condor, 66: 70-74.

Verbeek NAM. 1972. Daily and annual time budget of the Yellow-billed Magpie [J]. Auk, 89: 567-582.

Wang YH, Wu ZK, Li ZM, Jiang YM. 1989. Black-necked Crane and Study of China [J]. Zoological Research, 24(2): 35-39. [王有辉, 吴至康, 李 筑眉, 江亚猛. 1989. 我国的黑颈鹤与研究. 动物学杂志, 24(2): 35-39.]

Wu JD. 2005. Behavior study of wintering Siberian Cranes at Poyang Lake [A]. In: Wang QS, Li FS. Crane Research in China [M]. Kunming: Yunnan Educational Publishing House, 110-117. [吴建东. 2005. 白鹤 在鄱阳湖越冬期的行为研究. 见: 王岐山, 李风山. 中国鹤类研究. 昆明: 云南教育出版社, 110-117.]

Yang R, Wu HQ, Yang XJ, Jiang WG, Zuo L, Xiang ZR. 2007. Diurnal time budget of the black-necked crane during the breeding season [J]. Waterbirds, 30(1): 80-85.

Yang XJ, Qian FW, Li FS, Gao LB, Wu HQ. 2006. First satellite tracking of Black-necked Cranes in China [J]. Zool Res, 26(6): 657-658.[杨晓君， 钱法文, 李凤山, 高立波, 伍和启. 2006. 中国首次卫星跟踪黑颈鹤 研究初报. 动物学研究, 26(6): 657-658.]

Yang XJ, Yang L, Wang SZ, Wen XJ. 1998. The time budgets and activities of Derbys Parakeet in captive [J]. Acta Zoologica Sinica, 44(3):

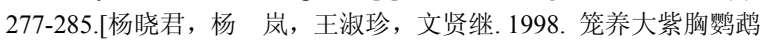
的活动时间分配. 动物学报, 44(3): 277-285.]

Yang XJ, Yang L. 1996. The observation of time budget of captive peafowl (Pavo muticus) [J]. Acta Zoologica Sinica, 42(suppl.): 106-111.[杨晓 君, 杨 岗. 1996. 笼养绿孔雀行为时间分配的初步观察. 动物学报, 42(增刊): 106-111.]

Yu YL, Liu WL, Sang J. 1993. A preminary study on the overwintering ecology of black-necked crane (Grus nigricollis) in the upper LHASA river [J]. Zool Res, 14(3): 250-251. [余玉群, 刘务林, 桑 杰. 1993. 拉 萨河上游黑颈鹤越冬生态的初步研究. 动物学研究, 14(3): 250-251.]

Zhang JJ, Luo J. 1991. Number and distribution of Black-necked Crane on Ruoergai Plateau Marsh [J]. Sichuan Journal of Zoology, 10(3): 37-38.[张家驹, 罗 佳. 1991. 若尔盖高原沼泽黑颈鹤数量分布. 四 川动物, 10(3): 37-38.]

Zhong XY, Dao MB. 2005. Cranes of the world [A]. In: Li FS, Yang XJ, Yang F. Status and conservation of Black-necked Cranes on the Yunnan and Guizhou Plateau, People' Republic of China [M]. Kunming: Yannan Nationalities Publishing House, 101-106. [钟兴耀, 道美标. 2005. 大山 包栖息地环境变化对越冬黑颈鹤的影响初探. 见: 李凤山, 杨晓君, 杨 芳. 云贵高原黑颈鹤的现状和保护. 昆明: 云南民族出版社, 101-106.] 